\title{
Surfactants Can be Modelled
}

\author{
DYNAMICAL PROCESSES IN MICELLES REPRODUCED
}

Amphiphilic molecules such as surfactants, lipids, or detergents have an affinity for both water and oil. The polar head of the molecule is hydrophilic, while the hydrocarbon chain is responsible for the affinity for oil. Dissolved in water, surfactants tend to aggregate in micelles. Micelles are simple forms of self assemblies. At higher surfactant concentration, various different assemblies such as bi-layer, cylindrical micelles, vesticles, and other structures may form. Despite the importance of these assemblies in various processes - ranging from the transport of molecules through upper-cell membranes to the removal of stains in a washing machine - our knowledge of self-assembly at a molecular level is still very poor. Computer simulations may contribute to a better understanding of these fascinating systems.

In our simulations we have used a simple water surfactant/model. The starting points of the model are: oil and water do not like each other, and a surfactant is an amphiphilic molecule, i.e., one side of the molecule likes oil but dislikes water, the other side likes water but dislikes oil. Both water and oil particles are modelled with a truncated Lennard-Jones potential. The truncation of the potential is made dependent on the type of interaction such that the oil-water interactions are purely repulsive and the waterwater and oil-oil interactions attractive. A surfactant is made up by several oil and water particles connected via harmonic springs
Snapshot of part of the surfactant/water system. For clarity only the surfactants are shown; the white spheres are the hydrophobic segments. A monolayer has formed at the interfaces of micelles in the water phase.

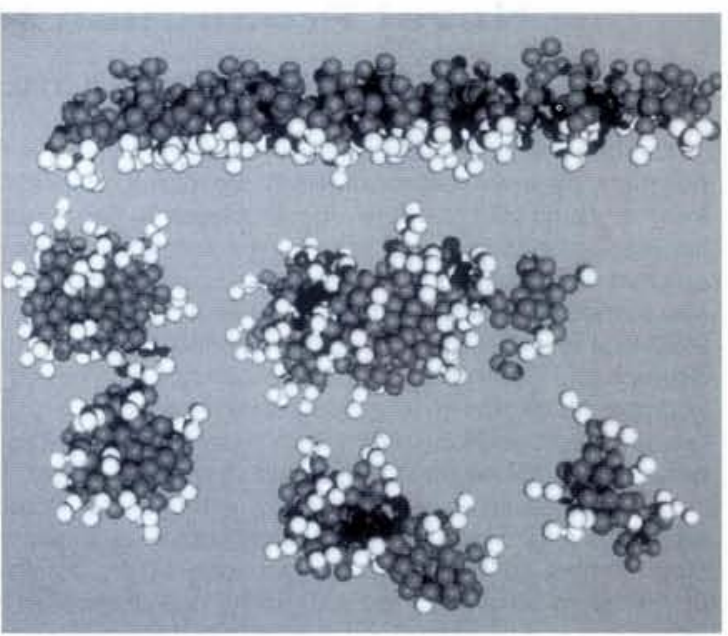

[Smit B., et al., Nature 348 (1990) 624; J. Phys. Chem. 95 (1991) 6361; Langmuir (in press) 1992]

Computer simulations on a water/surfactant system with 32000 particles were performed on a network of 400 transputers using a parallel molecular dynamics algorithm [Esselink, K., Smit, B., and Hilbers, P.A.J., J. Comp. Phys. (in press) 1992]. The simulations were started from a completely random distribution of surfactant. A snapshot of a small part of the equilibrated system is shown in the figure. It demonstrates that micelles have formed spontaneously.

One of the remarkable results of our simulations is that we can observe the dynamical processes in a micellar solution. The typical time scale of these processes have been determined experimentally. For example, the time scale for individual surfactants to leave a micelle is $10^{-8}$ to $10^{-6} \mathrm{~s}$, the fusion of two micelles takes $10^{-5}$ to $10^{-4} \mathrm{~s}$, and the typical lifetime of a micelle is of the order of $10^{-3}$ to $10^{-1} \mathrm{~s}$. These time scales are clearly (far) out of the range accessible by simulations on realistic models, where the maximum simulation time is of the order of $10^{-9} \mathrm{~s}$.

It turned out that our simple model does show these phenomena in a time span that is accessible in a computer simulation. This allows us to use molecular dynamics to study the dynamical processes that are of importance in many biological and industrial applications.

Shell Resear

lity with the increasing range of high-performance fibres and oriented polymers that are generally available in the market-place.

The Europhysics conference in St. Petersburg brought together the principal international investigators in this active field and provided a timely opportunity to review prospects and to look ahead. The chosen themes were wide-ranging, encompassing amorphous as well as crystalline systems, liquid as well as solid states, mechanisms of formation as well as resulting properties, understanding as well as knowledge. Due prominence was given to mechanical behaviour, but a notable session on conducting polymers, including a talk by $P$. Smith and U. Shirakawa brought much order into a particularly fast-moving area. It also emphasized that orientation improves with conductivity and mechanical behaviour in parallel, so that highly conductive systems tend to have good mechanical properties.

On mechanical aspects not only were the pioneers (Ward, Keller, Pennings, Lemstra, and others) present in person but each showed in their characteristic way the importance of current achievements and the rich potential the field still holds for the future (see box).

Scientifically, the conference was unquestionably first class, for the quality of its contributions, the discussion which was stimulated, and the personal contacts which were established and renewed. Logistically the conference was quite extraordinary. It had seemed to many that to organize any meeting in the circumstances of present-day Russia 


\section{Novel Production Methods}

\section{HOT COMPACTION OF POLYMERIC FIBRES}

The last twenty years have seen the development of high stiffness and high strength polymers by a number of different processing routes. The materials produced range from very high stiffness fibres (up to at least $90 \mathrm{GPa}$ ), manufactured by drawing isotropic fibres to a very high draw ratio, to thick-section products produced by pressing or pulling polymer through a die, whose stiffness is usually limited to $<40 \mathrm{GPa}$. There is therefore a requirement for high stiffness, thick-section materials. A process [British Patent: GB 2253420] for manufacturing such materials has been developed at the IRC in Polymer Science and Technology, Leeds University, UK. Termed "hot compaction", it takes highly drawn fibres and by choosing suitable conditions of temperature and pressure produces a homogeneous product which retains a high proportion of the original fibre properties while achieving a reasonable strength [J. Mater. Sci., to be published]

The important step was the discovery that it was possible to take a fibre below its melting range and selectively melt a small portion. Sufficient pressure needs to be applied to stop the fibre shrinking and losing its orientation, but not enough to inhibit melting. It is obviously an advantage to have the surface of the fibre melt first and anything that promotes this is desirable. For a hexagonal close-packed structure (a unidirectional arrangement of fibres) only $9 \%$ of the second phase is needed to fill all the holes and bind the structure, allowing substantial retention of fibre properties.

On cooling, the molten material recrystallises to form a second, lower melting point phase, clearly evident in differential scanning calorimetry. Morphological studies show the second phase to form a transcrystalline bridge between the fibres. The spaces between the fibres are completely filled with the melted and recrystallised polyethylene. Compaction is seen to be complete, and the fibres are in the main, still largely circular in cross-section.

Nucleation of the second phase is all along the fibre boundaries so that crystals grow out perpendicular to the fibres, meeting in the middle and forming a very strong bond. There is evidence from fracture studies that failure of the compacted composite occurs within the fibre itself, suggesting the melted and recrystallised material forms an excellent "glue", and that the weakness is within the fibre itself. This is confirmed by compaction studies on a range of fibres, which show the most highly aligned fibres (i.e., those with the lowest transverse properties) as having the lowest composite transverse strengths.

Fibres so far successfully compacted include melt spun, melt kneaded and gel-spun polyethylenes, polypropylene and PET. Other fibre configurations can be compacted including chopped fibres, woven fibre cloth and laminates of unidirectional fibres. All of these have improved strength compared to unidirectionally aligned fibres, but have lower stiffness. The compacted composites allow a light, tough and high stiffness composite to be manufactured using a single phase. This has considerable advantages over processing two-component composites.

P.J. Hine and I.M. Ward, IRC in Polymer Science \& Technology, Leeds, UK

R.H. Olley and D.C. Bassett, University of Reading, UK

\section{IBM RESEARCH}

\section{Shifting Emphasis to Remain Vital}

Dr. Karl Kümmerle, Director of the IBM Zurich Research Laboratory, discusses the impact on basic research of the company's "fundamental redefinition" announced last November, and the latest round of staff reductions.

IBM's Zurich Research Laboratory with a staff of about 200 represents a fairly small part of the Research Division (3300 staff and an annual budget of 600 M\$US which has remained almost constant for several years). It is dwarfed by IBM as a whole (300 000 employees; 69000 M\$US turn-over in 1991). Founded in 1956, established on the present site in 1962, expanded in the 1970's, modernised and expanded again in the mid-1980's, the Zurich lab, with its two Nobel prizes for discovering scanning tunnelling microscopy and high-temperature superconductivity, has acquired an outstanding reputation in basic physics research. Principal interests in physics and laser science are novel microscopies to the nanometre scale, high-Tc superconductors, numerically intensive computation,

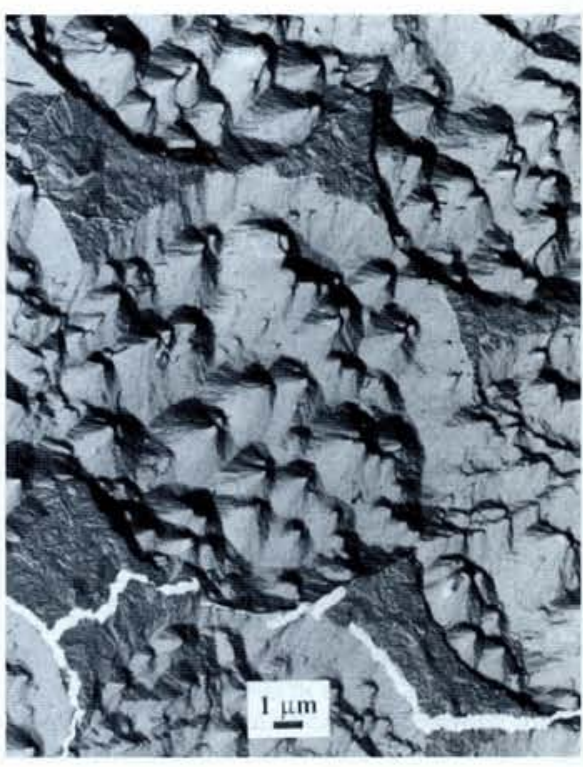

A photomicrograph, perpendicular to the main fibre direction, of an etched cross-section through hot-compacted polyethylene fibre.

would not be possible: but not to Professor V. Marikhin, Dr. L. Myasnikova and their colleagues. They accomplished the impossible and organized a Conference which was exceptional in every way. To attend and live in St. Petersburg in the season of the white nights was a great privilege for the twenty five western scientists who joined ninety Russian colleagues at this most pleasant and scientifically valuable occasion. The only cautionary note is that, in contrast to previous experience, very few attended from countries of the former eastern bloc: obtaining hard currency is the new barrier to attendance. This will need to be addressed to ensure that in future good science and scientists from all countries are able to attend Europhysics conferences. The next meeting is titled "Transitions in Oligomer and Polymer Systems" (Ulm, Germany; 27 September - 1 October 1993).

D.C. Bassett Chairman, EPS Macromolecular Board

ited number of "lightweight" partnerships which move quickly into a large number of niche markets, there is also a trend to form joint ventures with other companies to provide sufficient growth potential. There has been speculation in the press that a shift to applied research means that about 100 posts in basic research will go in the next few years.

Editor - One of IBM's goals was to be "famous for our science". Is this still the case? James McGroddy, your Director of Research, speaks of this science being a "vital" part of IBM. What does he mean?

Dr. Kümmerle - IBM has dual goals: it wants to be famous in science and technology and its science must also be relevant, in other words vital to IBM. The situation was established a long time ago and it is "burned in": it's part of the culture. We are famous by putting down stakes in selected fields as opposed to a "me too" strategy. The dual goal remains durable and nothing has changed in this respect. 